\title{
Cold Provocation and Active Thermography in Medical Screening
}

\author{
Michał Strzelecki ${ }^{1}$, Maria Strąkowska ${ }^{1}$, Robert Strąkowski ${ }^{1}$, Andrzej Kaszuba ${ }^{2}$ \\ ${ }^{1}$ Institute of Electronics, Lodz University of Technology \\ Wolczanska 211/215, 90-924 Lodz, Poland, \\ E-mail:michal.strzelecki@p.lodz.pl,maria.strakowska@dokt.p.lodz.pl,robert.strakowski@p.lodz.pl \\ ${ }^{2}$ Department of Dermatology, Pediatric Dermatology and Dermatological Oncology \\ Medical University of Lodz, Kniaziewicza 1/5, 91-347 Lodz, Poland \\ E-mail: andrzej.kaszuba@umed.lodz.pl
}

Received: 23 January 2017 ; accepted: 23 February 2017 ; published online: 23 March 2017

\begin{abstract}
This paper presents application of active thermography as a tool for supporting diagnosis of selected dermatological diseases. The cold stress method is used to stimulate human skin and to measure its temperature change in time. Image and data processing techniques are applied to calculate response parameters and plot their spatial distributions (maps). The proposed technique was applied to estimate differences between healthy and unhealthy tissue for patients with psoriasis. Another application concerns detection of regions characterized by different vascularization under the skin. Obtained preliminary results are promising. It was demonstrated that the developed method, after further testing, can be a useful tool to improve the standard diagnostic procedures.
\end{abstract}

Key words: active thermography, cold stress, temperature response, thermal skin parameter estimation

\section{INTRODUCTION}

One of the possible medical applications of thermography is dermatology, where this passive technique is implemented to measure temperature distribution on the surface of the human skin. There is a number of advantages of thermovision - it is contactless, non-invasive and, due to price reduction of temperature sensors, it has recently become significantly cheaper. However, the temperature distribution on the skin's surface results from individual thermal characteristics of the human body. In particular, this temperature depends on the energy balance inside the tissue, where both perfusion and blood flow in small and large vessels can be observed. Therefore, a more complex technique such as active thermography is used in order to evaluate thermal parameters in a quantitative way [1]. Thermal parameters characterizing the tissue are calculated from recorded temperature changes over time after thermal stimulation (cooling or warming) of the skin. The most popular approach is to fit the exponential curve to the recorded temperature variations [1]. The estimated time constants are then used to characterize the tissue. In order to approximate skin response more precisely the linear combination of exponential functions is also used [2-4]. To further improve this approximation, a combination of exponential and error functions (erfc) was used in this research. It was demonstrated that such approach approximates the temperature change more accurate [5] when the erfc term was added. Moreover, the considered model results from the physical multilayer structure of skin and heat flow [6]. It is assumed that estimated thermal parameters can be used to differentiate the state of the skin and inner tissue since the selected diseases, e.g. breast cancer (former research performed with standard thermography provided promising results [7]), inflammation, melanoma and other pathologies influence tissue vascularization [8-10].

Many of today's medical diagnosis techniques use imaging as the method for presenting the results of the human body assessment. It is one of the most convenient approaches 
to identify areas of interest where a further analysis should be undertaken. The results of active thermography in medical applications [11] can also be presented in the form of an image that shows maps representing spatial distribution of estimated parameters values.

The aim of this study is to characterize the key image processing techniques and to develop the software for application of active thermography as the medical imaging technique for supporting diagnosis of some dermatological diseases. The software was applied to estimate the differences between healthy and unhealthy tissue for patients with psoriasis. Cold stress was applied to the patient's skin and its thermal response was stored and analyzed as a function of time. The parameter values of an approximated thermal response were estimated and further used for differentiating between healthy and unhealthy parts of the skin. Another example of software application was analysis of the skin to detect blood vessels beneath it. Estimated thermal response parameter maps were used to visualize regions with different vascularization under the skin. Although the most commonly known method of veins patterns localization use near-infrared (NIR) spectrum, it can be used only for qualitative imaging [12, 13]. The method proposed in this paper also allows to characterize the blood vessels in a quantitative way by calculating their response parameters for thermal excitation.

The structure of this paper is as follows. Section 2 describes methods applied for thermogram processing and analysis to estimate thermal skin parameters and their maps. In Section 3 results obtained for psoriasis and vascularization studies are presented. Section 4 contains results discussion and concludes the paper.

\section{MATERIALS AND METHODS}

\section{1. Acquisition of thermograms}

The measurements were performed in the Biegański Provincial Specialist Hospital in Lodz on selected patients with psoriasis after obtaining their consent. Vascularization measurements were performed on healthy volunteers. In both cases MWIR (3-5 mm) Cedip Titanium camera with InSb cooled detector was used for thermograms acquisition. The obtained image resolution was $640 \times 512$ pixels and the camera had NETD $=17 \mathrm{mK}$. The images were recorded with a frame rate equal to 50 FPS and sequence mean acquisition time was 7 minutes.

In the case of the patients with psoriasis cold stress was applied on the same duration for healthy and unhealthy parts of the skin. If possible, both regions were located close to each other. In some cases, due to the progress of the disease, measurements were performed in other places. Experiments demonstrated that for psoriasis the most convenient cooling method is application of metal blocks (Fig. 1) placed on the skin for 6 seconds. It cooled the skin by about $5-7^{\circ} \mathrm{C}$ below its initial normal temperature. The regions of interest (ROIs), from which the data were extracted, were rectangular area $5 \times 5$ pixels around two points selected respectively in healthy and unhealthy part of the cooled skin.

The same cooling scheme was applied for vascularization study. The examination was performed according to the guidelines for thermovision measurements in medicine $[14,15]$. After acquisition of a sequence of infrared images, they were processed and analyzed using the developed software. In this case the ROIs were defined as the whole areas where the skin was cooled. Every pixel from these regions was processed independently.

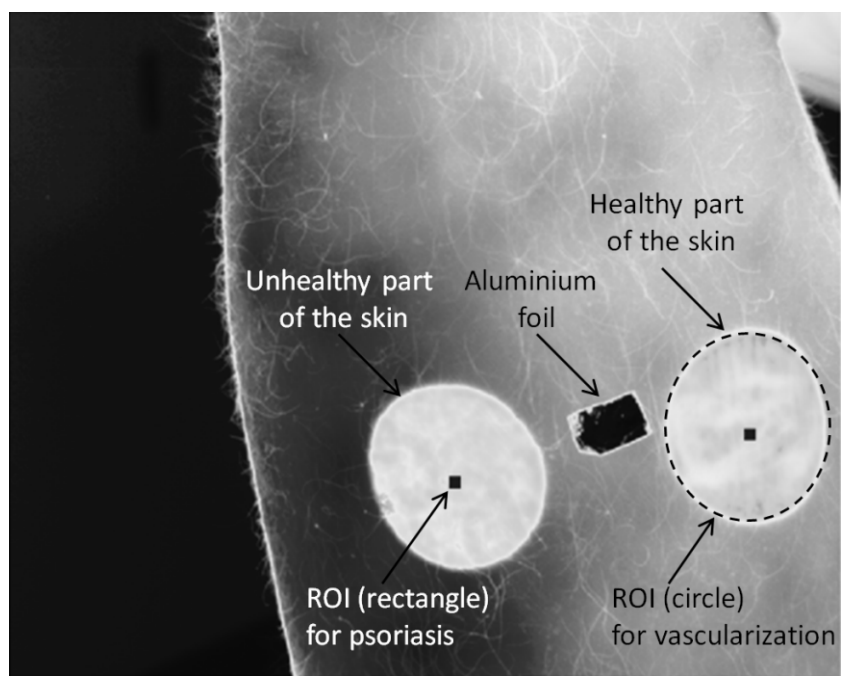

Fig. 1. Thermal image after cooling the patient's skin with aluminum foil stick on the skin surface and marked regions of interest

\section{2. Developed software}

Several steps should be performed to analyze and visualize the distribution of calculated model parameters from the skin thermal response. It is summarized in a diagram shown in Fig. 2. After thermogram acquisition, the first step is the movement correction. It is a necessary step in the measurement of a patient's skin temperature, as the recording lasts several minutes, during which patients breathe and slightly

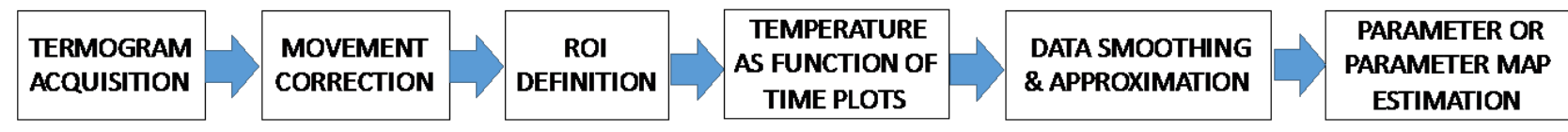

Fig. 2. Block diagram of developed thermogram analysis algorithm 


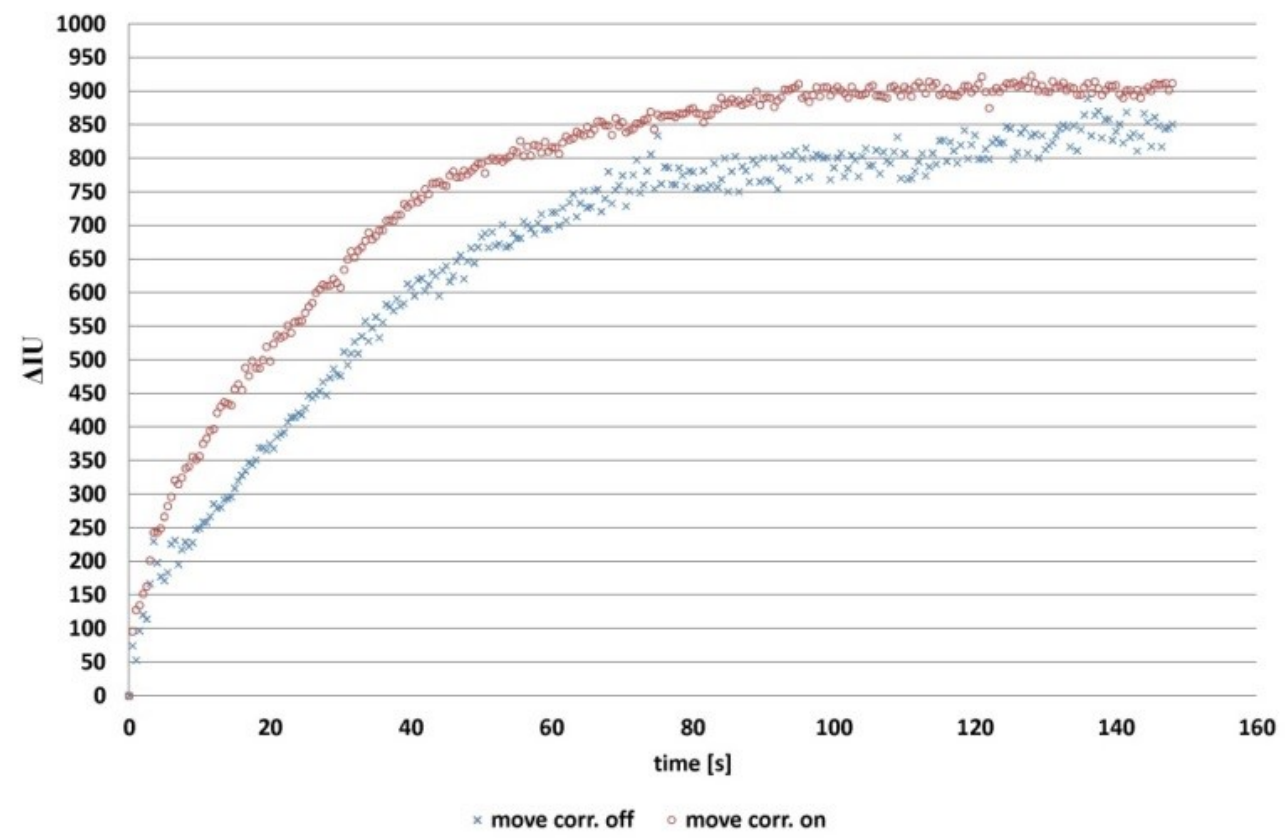

Fig. 3. Thermal response of the skin after cooling with and without movement correction [16]

move. Temperature change is examined for every point of the area under thermal stimulation.

All movements disturb the measurement and can cause wrong function approximation, leading to missing parameters calculation. Fig. 3 presents the plot of temperature rise (in Isothermal Units) as a function of time with and without the movement correction. The measurements were performed on the same region, but the obtained curves look completely different.

The movement correction is based on the cross-correlation method [16]. The method localizes the template image in the base image. The image position, which is most similar to the template, is indicated by the highest absolute value of the calculated cross-correlation coefficient. The output matrix of correlation coefficients $\gamma$, whose size is the same as the size of the base image, is calculated with the formula:

$$
\begin{aligned}
\gamma(u, v)= \\
=\frac{\sum_{x, y}\left(f(x, y)-\overline{f_{u, v}}\right)(t(x-u, y-v)-\bar{t})}{\sqrt{\sum_{x, y}\left(f(x, y)-\overline{f_{u, v}}\right)^{2}(t(x-u, y-v)-\bar{t})^{2}}}
\end{aligned}
$$

where $f(x, y)$ represents an image, $t$ is a template image, $\bar{t}$ is the mean of template, $\bar{f}$ is the mean of the image fragment covered by template. Details of the movement correction procedure are described in [18].
This method is sensitive for the intensity gradient in the image. In order to improve movement correction, the aluminum foil is placed on the body as a marker (see Fig. 1). It has different emissivity than human skin. This results in a difference in radiated power (despite the same temperature) as well as in pixel intensity in the recorded IR image. The algorithm tracks this marker (the template) and calculates its new position for every frame (the base image).

Based on the calculated data, the stabilized images in time are extracted from the recorded sequence. Briefly speaking, the algorithm works as the digital image stabilization technique performed after the recording process. Next, the region of interest (ROI) is determined where the temperature difference between the first and the last frame of the stabilized images are detected (the cooled areas). Afterwards, temperature change over time is extracted from the sequence of stabilized images. For parameter map evaluation, not all pixels in detected ROI are further processed because the final approximation of data curves is very time consuming. For example, the approximation of 4800 temperature curves, using MATLAB programming environment, takes around 50 minutes with Intel i7 microprocessor based PC. The whole ROI contains 19000 pixels. Therefore, the measured temperature is calculated as the mean value of $3 \times 3$ area for the pixels (centers of averaged area) that have coordinates with even columns and rows. As a result, only $\sim 17 \%$ of points from 
Tab. 1. The set of parameters for the healthy skin

\begin{tabular}{c|c|c|c|c|c|c}
\hline $\mathrm{T}$ (start) $\left[{ }^{\circ} \mathrm{C}\right]$ & $\mathrm{T}$ (end) $\left[{ }^{\circ} \mathrm{C}\right]$ & $\Delta T\left[{ }^{\circ} \mathrm{C}\right]$ & $A\left[{ }^{\circ} \mathrm{C}\right]$ & $B\left[{ }^{\circ} \mathrm{C}\right]$ & $\omega_{0}[1 / \mathrm{s}]$ & $\omega_{1}[1 / \mathrm{s}]$ \\
\hline 24.82 & 29.82 & 5.01 & 0.52 & 5.59 & 0.022 & 0.0260 \\
\hline 25.65 & 31.45 & 5.80 & 1.75 & 4.99 & 0.320 & 0.0220 \\
\hline 25.96 & 31.38 & 5.42 & 1.08 & 5.50 & 0.028 & 0.0298 \\
\hline
\end{tabular}

the region of interest are processed in the next steps, which allows to speed up the calculations. The extracted data often contain noise. It can be caused by incorrect movement correction or simply measurement noise. This noise is reduced with the use of a median filter followed by a mean filter. The filtration was applied for each pixel location separately in consequent frames. Thus it corresponds to one-dimensional filtration, where pixel values that change over time are analyzed. The number of frames considered (which corresponds to the length of filtration window) was $25(0.5 \mathrm{~s})$ for median and $200(8 \mathrm{~s})$ for mean filter, respectively. Finally, the approximation by the linear combination of exponential and error function (2) is applied.

$$
h(t)=A\left(1-e^{-\omega_{0} t}\right)+B\left(1-e^{\omega_{1} t} \operatorname{erfc}\left(\sqrt{\omega_{1} t}\right)\right)
$$

The approximation is done using the Pattern Search method available in MATLAB environment [17]. The final result is a set of 4 parameters: $A, B, \omega_{0}, \omega_{1}$ which are the parameters adjusted by the applied optimization procedure. Coefficients $A, B$ are the amplitudes and $\omega_{0}, \omega_{1}$ are the pulsations of curve components. In the case of parameter map estimation needed in vascularization study, the last step is to calculate parameters values for the pixels that were omitted during data extraction from the stabilized image. Parameters values for these points are interpolated as the mean values of the calculated ones in its nearest neighborhood.

\section{RESULTS}

\section{1. Psoriasis study}

The sample parameter values obtained from the measurements and approximation of the thermal response for psoriasis study are presented in Tables 1 and 2. Parameters $\left(A, B, \omega_{0}\right.$, $\omega_{1}$ ) estimated by means of the approximation function that contains a combination of the exponential and error function (2) were used to differentiate between the healthy and unhealthy parts of the skin. It is assumed that these parameters can be used as discriminating features and their values enable treatment effectiveness assessment of psoriasis disease.

The results show several regularities. The value of $\omega_{0}$ is lower for healthy part of the skin when compared to the

Tab. 2. The set of parameters for the unhealthy skin

\begin{tabular}{c|c|c|c|c|c|c}
\hline $\mathrm{T}$ (start) $\left[{ }^{\circ} \mathrm{C}\right]$ & $\mathrm{T}$ (end) $\left[{ }^{\circ} \mathrm{C}\right]$ & $\Delta T\left[{ }^{\circ} \mathrm{C}\right]$ & $\mathrm{A}\left[{ }^{\circ} \mathrm{C}\right]$ & $B\left[{ }^{\circ} \mathrm{C}\right]$ & $\omega_{0}[1 / \mathrm{s}]$ & $\omega_{1}[1 / \mathrm{s}]$ \\
\hline 24.46 & 28.99 & 4.53 & 0.38 & 5.23 & 0.311 & 0.022 \\
\hline 29.51 & 34.10 & 4.59 & 0.83 & 4.84 & 0.495 & 0.022 \\
\hline 27.90 & 32.60 & 4.46 & 1.04 & 4.25 & 0.348 & 0.023 \\
\hline
\end{tabular}

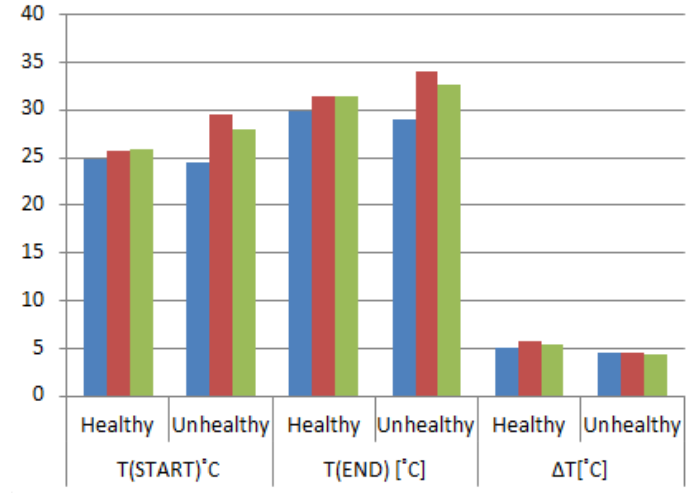

(a)

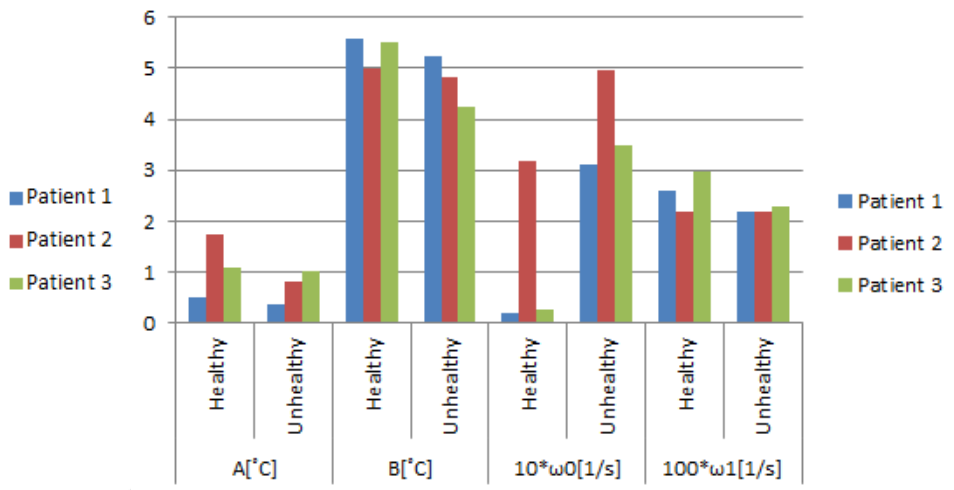

(b)

Fig. 4. Distribution of measured (a) and estimated (b) parameter values for sample patients [5] 
unhealthy. What can also be observed is the rising of skin temperature. The difference between final and initial temperature value is lower for unhealthy tissue. Also, parameters $A$ and $B$ have higher values for healthy tissue. This phenomenon is evident in Fig. 4. Such results are promising since skin classification seems to be possible; however, they should be confirmed by further measurements repeated for different patients.

\section{2. Vascularization study}

The preliminary tests for vascularization study were carried out on the forearm. The area where thermal stimulation was applied was carefully chosen. On the static infrared image, no veins were seen on the surface of the skin, as shown in Fig. 5. However, according to forearm anatomy, there are some veins distributed in the proximity of the skin surface. Thus blood flow underneath the skin surface, where the temperature is measured, should affect values of estimated thermal time constants. This assumption is confirmed in Fig. 6. One can notice the difference in temperature plots (both for raw and approximated data) obtained for the skin with and without blood vessels. It is also obvious that estimated time constants will differ for these two analyzed skin regions characterized by different vasculature.

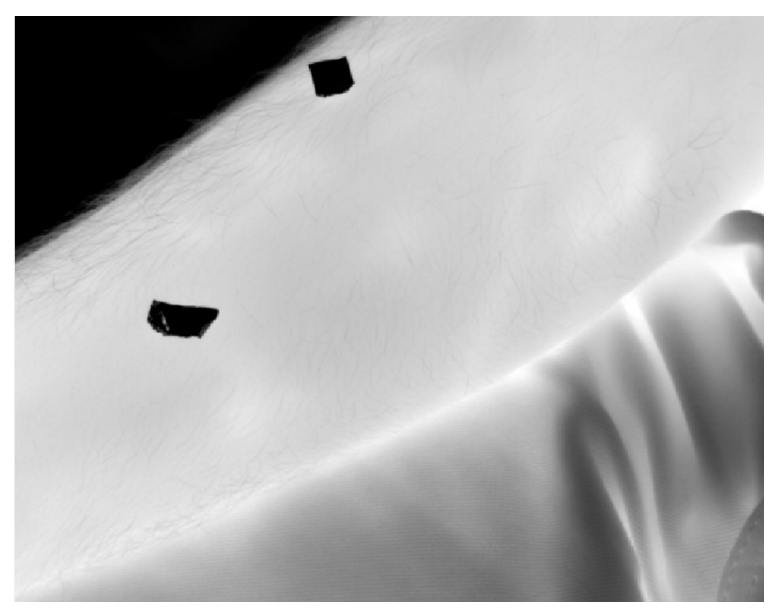

Fig. 5. Area under test before the examination aimed at vessel detection [18]

As s result of performed calculations, the spatial distribution of the values of four estimated parameters is visualized. All parameters are normalized and their values are coded with different gray levels. Prepared data are blended with the background image of recorded part of the human body to visualize the final effect more clearly. The blood vessels can be detected by analyzing estimated parameters maps. Unfortunately, some artifacts are also seen on the periphery of the selected ROI. Probably, this effect is caused by not fully precise movement correction. It is clearly seen that more information is carried by $\omega_{0}$ (Fig. 7a) and $\omega_{1}$ (Fig. 7b). Maps of $A$ and $B$ parameters are rather noisy thus do not contain any useful information about vessel distribution.

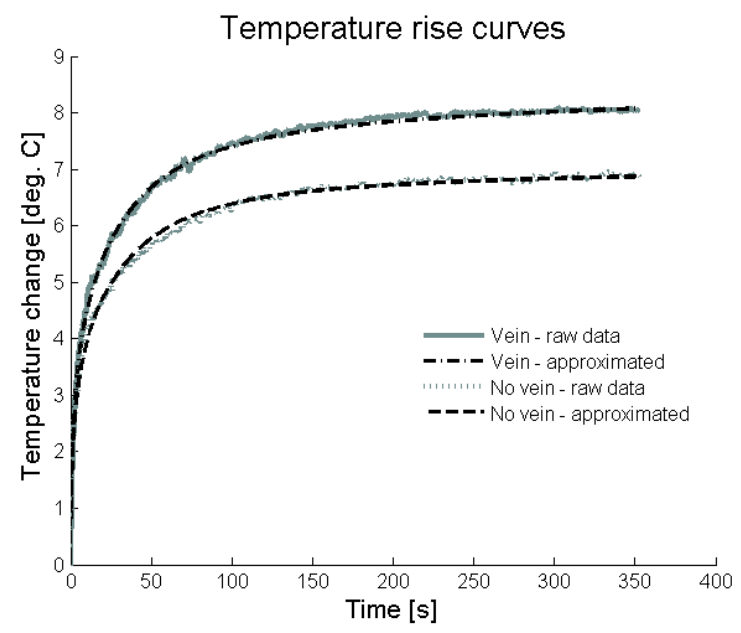

Fig. 6. Time plots of temperature rise after thermal stimulation, where: raw data are values of temperature as a function of time measured in sample point of interest, approximated data are curves obtained after approximation by Eq. (2) [18]

\section{DISCUSSION AND CONCLUSION}

The first results obtained using the proposed method of screening and processing the data to assess psoriasis were presented. Further research will focus on modeling the heat transfer in human skin and extracting the thermal parameters of the tissue. Based on these parameters as well as those obtained from the measurements the classification based on artificial intelligence tools will be applied. For example, it was demonstrated that neural networks (multilayer perceptron in particular) are efficient in classification of the aortic wall in echo images [19] and breast thermal images to detect pathological changes [20]. It is expected that the proposed approach will enable treatment monitoring and evaluation of its quality.
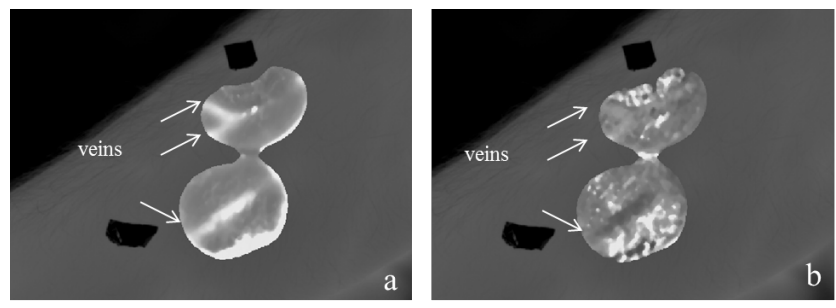

Fig. 7. Spatial distributions (maps) of parameters: $\omega_{0}(\mathrm{a}), \omega_{1}$ (b)

Also, preliminary results of the imaging technique based on active thermography and the linear combination of exponential and error functions [18] were presented. The proposed technique is based on estimation of spatial distributions of 
calculated curve parameters (maps). The discussed example confirms that this method is suitable for characterization skin areas with different vascularization. Based on this approach it can be assumed that the active thermography imaging can be a useful tool in the diagnosis of diseases that change the blood flow in affected areas of the tissue. One should remember that active thermography as the noninvasive and relatively chip technique is very attractive for practical diagnosis.

Further work will focus on the development of the presented software. At first, the movement correction module should be improved to eliminate artifacts on the periphery of the ROI. The approximation method of measured temperature rise should also be improved to remove noise in the visualization of spatial distribution of the selected parameters.

\section{References}

[1] A. Nowakowski Analiza technik diagnostycznych i terapeutycznych $w$ celu minimalizacji ryzyka interwencji kardiochirurgicznych, EXIT, Warszawa, (in Polish), (2008).

[2] T.M. Buzug, S. Schumann, L. Pfaffmann, U. Reinhold, J. Ruhlmann, Functional Infrared Imaging for Skin-Cancer Screening, EMBS Annual International Conference, New York City, USA, 2006.

[3] E. Laaperi, A-L. Laaperi, M. Strąkowska, B. Więcek, P. Przymusiała, Cold provocation improves breast cancer detection with IR thermography - A pilot study, Thermology International, ISSN-1560-604X, Volume 22, Number 4 (October), 152-156, 2012.

[4] D. K. Harrison, Thermal imaging method and apparatus, Patent EP 0885587 A1, 1998.

[5] M. Strąkowska, A. Kaszuba, M. Strzelecki Novel methodology of medical screening using IR thermography Signal Processing Algorithms, Architectures, Arrangements, and Applications - SPA 2014, Poznan.

[6] M. Strąkowska, G. De Mey, B. Wiecek, M. Strzelecki, A Three layer model for the thermal impedance of the human skin: modelling and experimental measurements, Journal of Mechanics in Medicine and Biology 15(3) (2015).

[7] T. Jakubowska, B. Wiecek, M. Wysocki, C. Drews-Peszynski, M. Strzelecki, Classification of breast thermal images using artificial neural networks, Proceedings of Annual International Conference of the IEEE Engineering in Medicine and Biology Society, San Francisco 2004, pp. 1155-1158.

[8] M. Kaczmarek, A. Nowakowski, Analysis of transient thermal processes for improved visualization of breast cancer using IR imaging. Engineering in Medicine and Biology Society, 2003. Proceedings of the 25th Annual International Conference of the IEEE. 2003;2:1113-1116.

[9] L. Weerd, JB. Mercer, S. Weum, Dynamic infrared thermography review article, Clinics in Plastic Surgery 38(2), 277-292 (2011).

[10] A. Mariotti, L. Di Carlo, G. Orlando, ML. Corradini, L/ Di Donato, P. Pompa, R. Iezzi, AR. Cotroneo, GL. Romani, A. Merla, Scrotal thermoregulatory model and assessment of the impairment of scrotal temperature control in varicocele. Ann. Biomed. Eng. 39(2), 664-73 (2011).

[11] A. Nowakowski, M. Kaczmarek, Active Dynamic Thermography - Problems of implementation in medical diagnostics, Quantitative InfraRed Thermography Journal 8(1), 89-106 (2011).

[12] N. Bouzida, A. Bendada, X. P. Maldague Observation of the human body thermoregulation and extraction of its vein signature using NIR and MWIR imaging, Proc. SPIE 7313, Smart Biomedical and Physiological Sensor Technology VI, April 24, 2009, DOI:10.1117/12.818285.

[13] S. Juric, B. Zalik, An innovative approach to near-infrared spectroscopy using a standard mobile device and its clinical application in the real-time visualization of peripheral veins, BMC Medical Informatics and Decision Making, 2014;14(1), DOI: $10.1186 / \mathrm{s} 12911-014-0100-\mathrm{z}$.

[14] EFJ. Ring, K. Ammer, The Technique of Infrared Imaging in Medicine, Thermology International 10(1) (2000).

[15] International Association of Certified Thermographers Standards \& Guidelines, http://www.iactthermography.org/stan dards_medical.html.

[16] M. Strąkowska, R. Strakowski, B. Wiecek, M. Strzelecki Cross-correlation based movement correction method for biomedical dynamic infrared imaging, 11th International Conference on Quantitative InfraRed Thermography, QIRT2012, 11-14 June 2012, Naples-Italy.

[17] C. Audet, J. E. Dennis Jr. Analysis of Generalized Pattern Searches SIAM Journal on Optimization 13(3), 889-903 (2003).

[18] M. Strąkowska, R. Strąkowski, M. Strzelecki, Thermal timeconstant imaging in cold-stress screening, Proc. of IEEE SPA 2015, 23-25 September 2015, Poznan, Poland, pp. 62-65.

[19] L. Chrzanowski, J. Drozdz, M. Strzelecki, M. KrzeminskaPakula, K. Jedrzejewski, J. Kasprzak, Application of neural networks for the analysis of histological and ultrasonic aortic wall appearance - an in-vitro tissue characterization study, Ultrasound in Medicine and Biology 34, 103-113 (2008).

[20] B. Więcek, M. Więcek, R. Strakowski, M. Strzelecki, T. Jakubowska, M. Wysocki, C. Drews-Peszyński. Breast cancer screening based on thermal image classification. Medical infrared imaging. Principles and practices, New York, 2013. 

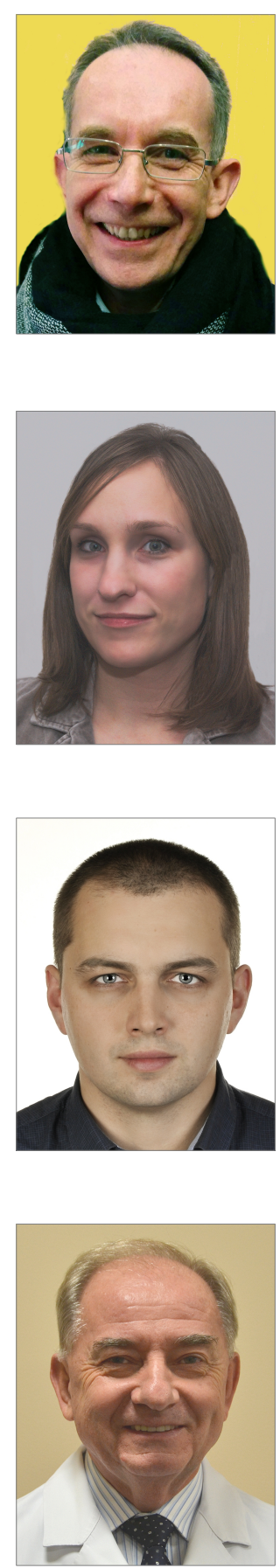

Michal Strzelecki (PhD 1995, DSc 2005) is at the Technical University of Lodz in Biomedical Engineering Division, Institute of Electronics (as Associate Professor since 2007). He worked as Visiting Professor in the Division of Electronics \& Information Engineering at Chonbuk National University in the Republic of Korea (2006 - 2008). His research interests are artificial neural networks, their hardware realizations, image processing and analysis, especially biomedical image texture segmentation, pattern recognition. He has also been involved in research concerning analog and mixed-signal circuit testing. Recently, he has been interested in networks of synchronized oscillators and their applications in analysis of biomedical images as well as in modeling of the human brain vascular system. Author or co-author of more than 200 scientific publications, he has participated in a number of international research projects including COST, European Union Framework Programme, etc. He is also a member of the Board of Directors of the European Campus Card Association and a member of the Association for Image Processing.

Maria Strąkowska was born in 1986. She has graduated from Lodz University of Technology (LUT), the Faculty of Electrical, Electronic, Computer and Control Engineering, obtaining her MSc in Electronics and Telecommunications with specialty in signal and image processing. Currently she is finishing her PhD thesis at the Department of Medical Electronics LUT. Her main research interest concerns the processing and analysis of thermal images and applications of thermography in various fields, particularly in medicine.

Robert Strąkowski was born in 1986. He has graduated from Lodz University of Technology, the Faculty of Electrical, Electronic, Computer and Control Engineering, obtaining his MSc in Electronics and Telecommunications with specialty in signal and image processing. In the Electronic Circuits and Thermography Division he conducts research on correction of the thermal drift in microbolometer thermal cameras. His research interests include the processing of thermal images and use of neural networks in data processing.

Andrzej Kaszuba (DSc 1993, Professor title 1998) is a head of the Department of Dermatology, Pediatric Dermatology and Oncology, Medical University of Lodz. Vice President of the Polish Society of Dermatology, chairman of the Lodz Division of the Polish Society of Dermatology, former National Consultant in the field of Dermatology and Venereology. Prof. Kaszuba is a supervisor of more than $30 \mathrm{Ph} . \mathrm{D}$. students in the field of medical science. He is author and co-author of 348 scientific papers published in highly ranked medical journals, 247 reports of conferences and scientific symposia organized in Poland and abroad, as well as 43 other scientific publications. 